\title{
Functional calculus for groups and applications to evolution equations
}

\author{
MARKUS HAASE
}

Abstract. Let $-i A$ be the generator of a $C_{0}$-group $U$ on a Banach space $X$. Via a transference principle we obtain results of the form

$$
\sup _{t \in \mathbb{R}}\|f(A+t)\|<\infty
$$

for certain functions $f$, provided that $X$ is a UMD space. Special examples are

$$
f(z)=\left(1+e^{z}\right)^{-1} \text { or } f(z)=\arctan z
$$

The first choice leads to easy proofs of the theorems of Monniaux and Dore-Venni, the second is related to a new proof of Fattorini's theorem on cosine functions.

\section{Introduction}

Over the last two decades it has become clear that many results of the theory of evolution equations can be read as actual results about certain types of functional calculi. For example, the setting of functional calculus allows to recover (and reduce to abstract principles) basic facts from evolution equations, like the Hille-Yosida and the Trotter-Kato theorems [8]. On a less elementary level, the abstract version of the maximal regularity problem can be framed as a question about the joint functional calculus for the two operators involved, see [7, Section 9.3] and [10]. Since for a long time this has been the main incentive, it was the functional calculus for sectorial operators which was in the focus of research. However, already in their famous 1987 paper [4] Dore and Venni used integrals over groups, namely the groups of imaginary powers $\left(A^{i s}\right)_{s \in \mathbb{R}}$. Since one expects to have

$$
f(A)=\int_{\mathbb{R}} g(s) A^{i s} d s \quad \text { if } \quad f(z)=\int_{\mathbb{R}} g(s) z^{i s} d s \quad\left(z \in S_{\theta}\right) .
$$

at least for certain functions $f$, the Mellin transform entered the scene.

Now, the generator of the group $\left(A^{i s}\right)_{s \in \mathbb{R}}$ is the operator logarithm $\log (A)$, hence it was sensible to study this operator in its own right. To this aim the so-called strip-type operators (with $\log (A)$ being the prototype example) were introduced in [6], together with

Mathematics Subject Classifications (2000): 47A60, 47D06.

Key words: $C_{0}$-semigroup, group, functional calculus, UMD space, Monniaux's theorem, Dore-Venni theorem, maximal regularity, Fattorini's theorem, cosine function. 
a natural functional calculus and suitable composition rules. It turned out that - for sectorial operators - the two points of view (sector or strip) are interchangeable. However, the strip situation is more general: every injective sectorial operator has a logarithm (which is striptype), but not every strip-type operator is the logarithm of a sectorial one. Therefore, the famous theorem of McIntosh [12] about functional calculus for sectorial operators on Hilbert spaces is a consequence of the Boyadzhiev-de Laubenfels theorem from [3] (see also [7, Theorem 7.2.1] and [9]) about functional calculus on strips, but not vice versa. Monniaux posed the question, which groups are actually groups of imaginary powers, and she gave a stunning (although partial) answer in [13]. Phrased differently, it is exactly the question, which strip-type operators are logarithms. But instead of setting up a functional calculus for the group generator, she defined the sectorial operator by means of the notion of analytic generator. Uiterdijk in [17] recast her work into a functional calculus framework, but not for the group generator itself but for the analytic generator. So again the Mellin transform was all over the place, and one is moreover in the strange situation that a functional calculus is constructed for an operator that is still to be found, and in the general case is weird anyway. (It can happen that the exponential $e^{A}$ of a strip-type operator $A$ has empty resolvent.)

Now, what is the major benefit of having a group in the first place? It lies in the fact that one can exploit a certain geometrical condition on the Banach space (called the UMD condition) to make converge certain singular integrals over the group. More precisely, if $U=(U(s))_{s \in \mathbb{R}}$ is a group on a UMD space $X$, the principal value integral

$$
T x:=\mathrm{PV}-\int_{-1}^{1} U(s) x \frac{d s}{s}
$$

exists for every $x \in X$, and one has a bound $\|T x\| \leq C \sup _{s \in(-2,2)}\|U(s)\|\|x\|$, where $C$ does not depend on the group but only on the space $X$. The main idea was present already in the Dore-Venni paper, and Monniaux in [13] made it explicit. However, the functional calculus interpretation of these results was still hidden, and it is one of the purposes of the present paper to change this.

The UMD property plays a role also in several other results in abstract evolution equations, e.g. in the result about the complex inversion formula [1, Section 3.12] and Fattorini's theorem on cosine functions [1, Theorem 3.16.7]. The latter theorem says that for a cosine function on a UMD space the first component of the phase space is essentially the same as the domain of the square root of the generator. It is our second major aim to provide a new proof of Fattorini's theorem by showing that it is nothing else than a result about functional calculus.

The paper is organised as follows. Departing from the functional calculus for strip-type operators, we turn to generators of groups and the so-called Phillips calculus. Then we prove an easy transference principle (Theorem 3.1) which allows us to bring in the UMD property (Theorem 3.6). In Section 4 we give a new proof of Monniaux's theorem and the Dore-Venni theorem. Since we can work with the groups directly, we neither need analytic 
generators nor the Mellin transform. We can work directly with the Fourier transform, which is much more convenient and apparently more natural. In Section 5 we give a new proof of Fattorini's theorem, based on the functional calculus for groups. We show that Fattorini's theorem is related to the fact that $\arctan (A)$ is a bounded operator if $-i A$ generates a $C_{0}$-group of type $<1$ on a UMD space $X$. Section 6 is an appendix, where we provide certain facts about the group reduction of a cosine function and a simple composition rule; these are interesting in their own right.

Let us introduce some notation. We usually consider (unbounded) closed operators $A, B$ on a Banach space $X$. By $\mathcal{L}(X)$ we denote the set of all bounded (fully-defined) operators on $X$. The domain and the range of a general operator $A$ are denoted by $\mathcal{D}(A)$ and $\mathcal{R}(\mathcal{A})$, respectively. Its resolvent is $R(\lambda, A)=(\lambda-A)^{-1}$, and $\varrho(A)$ denotes the set of $\lambda \subset \mathbb{C}$ where $R(\lambda, A) \in \mathcal{L}(X)$. Its complement $\sigma(A)=\mathbb{C} \backslash \varrho(A)$ is the spectrum. For given $\omega>0$ we define

$$
S t_{\omega}:=\{z \in \mathbb{C}|| \operatorname{Im} z \mid<\omega\}
$$

to be the horizontal strip of height $2 \omega$, symmetric about the real axis. We write $H^{\infty}\left(S t_{\omega}\right)$ for the Banach algebra of bounded holomorphic functions on $S t_{\omega}$. The set of complex Borel measures on a locally compact space $\Omega$ is denoted by $\mathbf{M}(\Omega)$. The Fourier transform of a tempered distribution $\Phi$ on $\mathbb{R}$ is denoted by $\mathcal{F}(\Phi)$ or $\widehat{\Phi}$. We often write $s$ and $t$ (in the Fourier image) to denote the real coordinate, e.g. $\sin t / t$ denotes the function $t \mapsto \sin t / t$.

\section{Functional calculus prelims}

Let $-i A$ be the generator of a $C_{0}$-group $(U(s))_{s \in \mathbb{R}}$ on a Banach space $X$. It is an elementary fact that there exist constants $M \geq 1, \omega \geq 0$ such that $\|U(s)\| \leq M e^{\omega|s|}$, $s \in \mathbb{R}$. The infimum of all such $\omega \geq 0$ is called the group type of $U$ and is (here) denoted by $\theta(U)$. General semigroup theory yields that the resolvent of $A$ satisfies the estimate

$$
\|R(\lambda, A)\| \leq \frac{M}{|\operatorname{Im} \lambda|-\omega} \quad(|\operatorname{Im} \lambda|>\omega),
$$

hence $A$ is a so-called strong strip-type operator of height $\omega_{s s t}(A) \leq \omega$, as defined in [7, Section 4.1]. There is a natural holomorphic functional calculus associated with operators of such a kind: in a first step one uses the Cauchy formula to define

$$
f(A):=\frac{1}{2 \pi i} \int_{\Gamma} f(z) R(z, A) d z
$$

where $f$ is a holomorphic function on a strip in the class $\mathcal{E}(\theta)$ defined by

$$
\mathcal{E}(\theta):=\left\{f \in H^{\infty}\left(S t_{\theta}\right) \mid \exists \varepsilon>0: f(z)=O\left(|z|^{-(1+\varepsilon)}\right)(|\operatorname{Re} z| \rightarrow \infty)\right\},
$$


for some $\theta>\omega$. The contour $\Gamma$ is the positively oriented boundary of a smaller strip $S t_{\omega^{\prime}}$, with $\omega^{\prime} \in(\omega, \theta)$ being arbitrary. This yields an algebra homomorphism of the algebra $\mathcal{E}(\theta)$ into the algebra of bounded operators on $X$. In a second step, by so-called regularisation, one defines $f(A)$ for a much wider class of functions:

$$
f(A):=e(A)^{-1}(e f)(A),
$$

where $e \in \mathcal{E}(\theta)$ is such that also ef $\in \mathcal{E}(\theta)$ and $e(A)$ is injective. The function $e$ is called a regulariser for $f$, and the definition of $f(A)$ is independent of the chosen regulariser. For example, if $f \in H^{\infty}\left(S t_{\theta}\right)$, then $f$ is regularisable by any function $e(z)=(\lambda-z)^{-2}$, where $|\operatorname{Im} \lambda|>\theta$. Details of the construction as well as a listing of all the formal properties of the so constructed functional calculus can be found in [7, Chapter 1 and 4].

Of particular importance in the theory of functional calculus is the so-called convergence lemma. We state and prove it here for the convenience of the reader.

PROPOSITION 2.1. (Convergence Lemma) Let A be a strong strip-type operator on the Banach space $X$, with dense domain. Let $\theta>\omega_{\text {sst }}(A)$ and let $\left(f_{l}\right)_{l \in \mathcal{J}}$ be a net of holomorphic functions on the strip $S t_{\theta}$, satisfying

1) $\sup \left\{\left|f_{\iota}(z)\right| \mid z \in S t_{\theta}, \iota \in \mathcal{J}\right\}<\infty$;

2) $f_{\iota}(z) \rightarrow f(z)$ for every $z \in S t_{\theta}$;

3) $\sup _{\iota}\left\|f_{\iota}(A)\right\|<\infty$.

Then $f(A) \in \mathcal{L}(X)$ and $f_{l}(A) \rightarrow f(A)$ strongly.

Proof. By Vitali's theorem, conditions (1) and (2) imply that $f$ is holomorphic, so $f(A)$ is defined. Moreover, $f_{\iota} \rightarrow f$ uniformly on compacts. Choose any $\lambda$ with $|\operatorname{Im} \lambda|>\theta$ and define $e(z)=(\lambda-z)^{-2}$. By some version of Lebesgue's theorem, $\left(e f_{l}\right)(A) \rightarrow(e f)(A)$ in norm. This implies $f_{l}(A) x \rightarrow f(A) x$ for all $x \in \mathcal{D}\left(A^{2}\right)$. Condition (3) now implies that $f(A)$ is a bounded operator, and since $\mathcal{D}\left(A^{2}\right)$ is dense in $X$, we have $f_{l}(A) x \rightarrow f(A) x$ for all $x \in X$.

Up to now we have not used our assumption that $-i A$ generates a $C_{0}$-group. This additional assumption provides us with a convenient tool to identify functions $f$ such that $f(A)$ is a bounded operator. First of all, consider for $s \in \mathbb{R}$ the function $e^{-i s z}$, which is bounded on every horizontal strip. We clearly expect $e^{-i s z}(A)=U(s)$ for all $s \in \mathbb{R}$. More generally, let $\omega \geq 0$ be fixed such that $\|U(s)\| \leq M e^{\omega|s|}$ for some $M \geq 1$ and all $s \in \mathbb{R}$, and let $\mu$ be a (complex) Borel measure on $\mathbb{R}$ satisfying

$$
\|\mu\|_{\mathbf{M}_{\omega}}:=\int_{\mathbb{R}} e^{\omega|s|}|\mu|(d s)<\infty .
$$


Then one can set

$$
T_{\mu} x:=\int_{\mathbb{R}} U(s) x \mu(d s) \quad(x \in X) .
$$

Clearly, the set $\mathbf{M}_{\omega}(\mathbb{R}):=\{\mu \mid(2.1)$ holds $\}$ is a Banach algebra with respect to convolution, and the map $\left(\mu \longmapsto T_{\mu}\right): \mathbf{M}_{\omega}(\mathbb{R}) \longrightarrow \mathcal{L}(X)$ is a homomorphism of algebras, called the Phillips calculus. Of course we expect $T_{\mu}=f(A)$ where $f$ is the Fourier-Stieltjes transform

$$
f(z)=\widehat{\mu}(z):=\int_{\mathbb{R}} e^{-i s z} \mu(d s) \quad\left(z \in S t_{\theta}\right)
$$

of $\mu$. Note that $\widehat{\mu} \in H^{\infty}\left(S t_{\omega}\right) \cap \mathbf{C}^{\mathbf{b}}\left(\overline{S t_{\omega}}\right)$. Here is the precise result.

LEMMA 2.2. Let $X, A$, and $U$ be as above, and let $\theta>\omega$.

a) Each function $f \in \mathcal{E}(\theta)$ arises as a Fourier-Stieltjes transform, namely

$$
f=\widehat{g} \text { with } g(s):=\frac{1}{2 \pi} \int_{\mathbb{R}} f(t) e^{i s t} d t \quad(s \in \mathbb{R}) .
$$

One has $g \in \mathbf{C}_{\mathbf{0}}(\mathbb{R}) \cap \mathbf{C}^{\frac{1}{2}}(\mathbb{R})$ and $\int|g(s)| e^{\alpha|s|} d s<\infty$ for all $\alpha \in[0, \theta)$; in particular, one has $g(t) d t \in \mathbf{M}_{\omega}(\mathbb{R})$.

b) Let $\mu \in \mathbf{M}_{\omega}(\mathbb{R})$, and suppose that $f:=\widehat{\mu}$ extends to a holomorphic function on $S t_{\theta}$ such that $f(A)$ is defined. Then $f(A)=T_{\mu} \in \mathcal{L}(X)$ and

$$
\sup _{t \in \mathbb{R}}\|f(t+A)\| \leq M\|\mu\|_{\mathbf{M}_{\omega}} .
$$

Proof. Part a) is essentially standard. For the Hölder continuity note that if $f \in \mathcal{E}(\theta)$ then $f(t)$ and $t f(t)$ are $\mathbf{L}^{\mathbf{2}}$-functions on $\mathbb{R}$, hence by Plancherel's theorem $g \in \mathbf{W}^{\mathbf{1 , 2}}(\mathbb{R})$. The embedding $\mathbf{W}^{\mathbf{1 , 2}}(\mathbb{R}) \subset \mathbf{C}_{\mathbf{0}}(\mathbb{R}) \cap \mathbf{C}^{\frac{1}{2}}(\mathbb{R})$ is a well-known (easy) Sobolev embedding.

To prove part b), suppose first that $f=\widehat{g} \in \mathcal{E}(\theta)$. Then we choose $\alpha \in(\omega, \theta)$ and compute

$$
\begin{aligned}
f(A) & =\frac{1}{2 \pi i} \int_{|\operatorname{Im} z|=\alpha} f(z) R(z, A) d z \\
& =\frac{1}{2 \pi i} \int_{\mathbb{R}-i \alpha} f(z) R(z, A) d z-\frac{1}{2 \pi i} \int_{\mathbb{R}+i \alpha} f(z) R(z, A) d z \\
& =\frac{1}{2 \pi} \int_{\mathbb{R}-i \alpha} f(z) R(i z, i A) d z+\frac{1}{2 \pi} \int_{\mathbb{R}+i \alpha} f(z) R(-i z,-i A) d z
\end{aligned}
$$




$$
\begin{aligned}
& =\frac{1}{2 \pi} \int_{\mathbb{R}-i \alpha} f(z) \int_{0}^{\infty} e^{-i z s} U(-s) d s d z+\frac{1}{2 \pi} \int_{\mathbb{R}+i \alpha} f(z) \int_{0}^{\infty} e^{i s z} U(s) d s d z \\
& =\frac{1}{2 \pi} \int_{-\infty}^{0} \int_{\mathbb{R}-i \alpha} f(z) e^{i s z} d z U(s) d s+\frac{1}{2 \pi} \int_{0}^{\infty} \int_{\mathbb{R}+i \alpha} f(z) e^{i s z} d z U(s) d s .
\end{aligned}
$$

We now shift the contour in the inner integrals and obtain

$$
\begin{aligned}
\ldots & =\frac{1}{2 \pi} \int_{-\infty}^{0} \int_{\mathbb{R}} f(z) e^{i s z} d z U(s) d s+\frac{1}{2 \pi} \int_{0}^{\infty} \int_{\mathbb{R}} f(z) e^{i s z} d z U(s) d s \\
& =\int_{-\infty}^{\infty} \frac{1}{2 \pi} \int_{\mathbb{R}} f(t) e^{i s t} d t U(s) d s=\int_{-\infty}^{\infty} g(s) U(s) d s .
\end{aligned}
$$

For a general $\mu \in \mathbf{M}_{\omega}(\mathbb{R})$, let $f:=\widehat{\mu}$ be its Fourier transform and let $e \in \mathcal{E}(\theta)$ be any regulariser of $f$, i.e., ef $\in \mathcal{E}(\theta)$ and $e(A)$ is injective. By a) we can pick functions $g, h$ such that $\widehat{g}=e$ and $\widehat{h}=e f=\widehat{g} \widehat{\mu}=(g * \mu) \widehat{\text {. Hence }} h=g * \mu$, and therefore

$$
(e f)(A)=T_{h}=T_{g * \mu}=T_{g} T_{\mu}=e(A) T_{\mu} .
$$

This implies that indeed $T_{\mu}=e(A)^{-1}(e f)(A)=f(A)$, as it was to be shown.

The underlying principle here is that because already certain functions $f$ (here: the exponentials $e^{-i t z}$ ) are assumed to lead to bounded operators $f(A)$, averaging over these yields again such functions. In the next section we shall see that in UMD spaces one can go a step further. But before, we turn to the "universal counterexample".

\subsection{The universal counterexample}

Fix $\omega \geq 0$ and let

$$
X:=\mathbf{L}_{\omega}^{1}(\mathbb{R}):=\left\{f \in \mathbf{L}_{\mathrm{loc}}^{1}(\mathbb{R}) \mid \int_{\mathbb{R}} f(t) e^{\omega|t|} d t<\infty\right\} .
$$

Consider on $X$ the translation group $U$ defined by $[U(s) f](t)=f(t+s)$. Then it is easy to see that $\|U(s)\|=e^{\omega|s|}, s \in \mathbb{R}$. The generator $-i A$ of $U$ is of course the derivative operator $d / d t$, with its natural domain, cf. [7, Section 8.4]. Let $\mu \in \mathbf{M}_{\omega}(\mathbb{R})$. Then it is easily seen that

$$
T_{\mu} f=\mu^{\sim} * f \quad(f \in X),
$$


where $\mu^{\sim}(B)=\mu(-B)$ for every Borel set $B \subset \mathbb{R}$. It is straightforward to show that $\left\|T_{\mu} f\right\| \leq\|\mu\|_{\mathbf{M}_{\omega}(\mathbb{R})}\|f\|_{X}$. However, we claim that actually

$$
\left\|T_{\mu}\right\|_{\mathcal{L}(X)}=\|\mu\|_{\mathbf{M}_{\omega}} .
$$

To establish this, take $\phi, \psi \in \mathbf{C}_{\mathbf{c}}(\mathbb{R})$ and compute

$$
\begin{aligned}
\left|\left\langle\mu^{\sim} * \psi, \phi\right\rangle\right| & =\left|\int \phi(t) \int \psi(t+s) \mu(d s) d t\right|=\left|\int \psi(t) \int \phi(t-s) \mu(d s) d t\right| \\
& =\left|\int \psi(t) e^{\omega|t|} e^{-\omega|t|} \int \phi(t-s) \mu(d s) d t\right| .
\end{aligned}
$$

Taking the supremum over the $\psi$ such that $\|\psi\|_{X} \leq 1$ yields

$$
\sup _{\psi}\left|\left\langle\mu^{\sim} * \psi, \phi\right\rangle\right|=\sup _{t \in \mathbb{R}}\left|e^{-\omega|t|} \int \phi(t-s) \mu(d s)\right| \geq\left|\int \phi(-s) \mu(d s)\right| .
$$

Taking the supremum over the $\phi$ such that $\sup _{s \in \mathbb{R}}\left|\phi(-s) e^{-\omega|s|}\right| \leq 1$ finally yields $\left\|T_{\mu}\right\| \geq$ $\|\mu\|_{\mathbf{M}_{\omega}(\mathbb{R})}$.

PROPOSITION 2.3. Let $0 \leq \omega<\theta$ and let $f \in H^{\infty}\left(S t_{\theta}\right)$. Then the following assertions are equivalent:

(i) $f(A)$ is a bounded operator, for every operator $A$ on a Banach space $X$ such that $-i$ A generates a $C_{0}$-group with $\|U(s)\| \leq M e^{\omega|s|}$ for some $M \geq 1$ and all $s \in \mathbb{R}$.

(ii) $f(A)$ is a bounded operator, where $X=\mathbf{L}_{\omega}^{1}(\mathbb{R})$ and $A=i d / d t$ with maximal domain.

(iii) $f=\widehat{\mu}$ for some $\mu \in \mathbf{M}_{\omega}(\mathbb{R})$.

Proof. Clearly (i) implies (ii), and the implication (iii) $\Rightarrow$ (i) is just Lemma 2.2. Suppose that (ii) holds true. Consider $e_{n}(z):=\left[n(n+i z)^{-1}\right]^{2} \in \mathcal{E}\left(S t_{\theta}\right)$ (for $n \in \mathbb{N}$ large enough). One has $e_{n}(A)=[n R(n,-i A)]^{2} \rightarrow I$ strongly as $n \rightarrow \infty$. Since $f(A) \in \mathcal{L}(X)$, $\left(e_{n} f\right)(A)=f(A) e_{n}(A) \rightarrow f(A)$ strongly. By Lemma 2.2 one finds $g_{n} \in \mathbf{L}_{\omega}^{1}(\mathbb{R})$ such that $\widehat{g_{n}}=e_{n} f$. Because of (2.2),

$$
\sup _{n}\left\|g_{n}\right\|_{\mathbf{L}_{\omega}^{1}}=\sup _{n}\left\|\left(e_{n} f\right)(A)\right\|_{\mathcal{L}(X)}<\infty .
$$

Now, $\mathbf{L}_{\omega}^{1}(\mathbb{R})$ embeds isometrically into $\mathbf{M}_{\omega}(\mathbb{R})$, which is the dual of the space

$$
\left\{f: \mathbb{R} \longrightarrow \mathbb{C} \mid f(t) e^{-\omega|t|} \in \mathbf{C}_{\mathbf{0}}(\mathbb{R})\right\}
$$

by the canonical duality. Hence there exists a subsequence $\left(g_{n_{k}}\right)_{k}$ that is convergent to some $\mu \in \mathbf{M}_{\omega}(\mathbb{R})$ in the weak* topology. Embedding everything into the space of tempered distributions on $\mathbb{R}$, it follows easily that $\widehat{\mu}=f$. (Note that the Fourier transform is weakly continuous on tempered distributions.) 
The previous proposition explains why the shift group on $\mathbf{L}^{1}$ is a universal counterexample: if there is at all an example of an operator $A$ where - for a given function $f-f(A)$ is not bounded, then already $f(d / d t)$ on $\mathbf{L}_{\omega}^{1}(\mathbb{R})$ is not bounded. Since one knows something about Fourier transforms of measures, Proposition 2.3 puts some obvious restrictions on functions $f$ in order that $f(A)$ is bounded in general. E.g., it is necessary that $f=\widehat{\mu}$ has Cesaro-limits at $\pm \infty$, and these limits have to be equal to $\mu\{0\}$, see [7, Proposition E.4.3]. In particular, if $a_{+}:=\lim _{\operatorname{Re} z \rightarrow \infty} f(z)$ and $a_{-}:=\lim _{\operatorname{Re} z \rightarrow-\infty} f(z)$ exist, then $a_{+}=a_{-}$. Therefore, functions $f$ like

$$
f(z)=\arctan z \quad \text { or } \quad f(z)=\frac{1}{1+e^{z}}
$$

do not lead to bounded operators in general, and the counterexample is always the shift group on (the weighted) $\mathbf{L}^{1}$-space.

\section{A simple transference principle}

Let $-i A$ be the generator of a group $(U(s))_{s \in \mathbb{R}}$ on a Banach space $X$, let $\mu \in \mathbf{M}[-1,1]$ and let $f:=\widehat{\mu}$. Then

$$
f(A)=T_{\mu} x=\int_{-1}^{1} U(s) x \mu(d s)
$$

as was seen above. Denote by

$$
L_{\mu}:=(f \mapsto f * \mu): \mathbf{L}^{p}(\mathbb{R} ; X) \rightarrow \mathbf{L}^{p}(\mathbb{R} ; X)
$$

the convolution operator on the $X$-valued $\mathbf{L}^{p}$-space. Transference means the fact that a good estimate of the operator $L_{\mu}$ implies a good estimate for the operator $T_{\mu}$.

THEOREM 3.1. Let $U$ be a $C_{0}$-group on a Banach space $X$, let $p \in[1, \infty)$ and set $M:=\sup _{-2 \leq s \leq 2}\|U(s)\|$. Then

$$
\left\|\int_{[-1,1]} U(s) x \mu(d s)\right\| \leq 2^{1 / p} M^{2}\left\|L_{\mu}\right\|_{\mathcal{L}\left(\mathbf{L}^{p}(\mathbb{R} ; X)\right)}\|x\| \quad(x \in X)
$$

for all $\mu \in \mathbf{M}[-1,1]$.

Proof. Let $|t| \leq 1$. Then we write

$$
T_{\mu} x=\int_{-1}^{1} U(s) x \mu(d s)=U(t) \int_{-1}^{1} U(s-t) x \mu(d s)=U(t)(f * \mu)(t)
$$


where $f(s)=\mathbf{1}_{[-2,2]}(s) U(-s) x$. Hence

$$
\left\|T_{\mu} x\right\|=\left\|\frac{1}{2} \int_{-1}^{1} U(t)(f * \mu)(t) d t\right\| \leq 2^{\frac{1}{p^{\prime}}-1} M\|f * \mu\|_{\mathbf{L}^{p}(R ; X)} \leq 2^{\frac{1}{p}} M^{2}\left\|L_{\mu}\right\|\|x\|
$$

where $\left\|L_{\mu}\right\|=\left\|L_{\mu}\right\|_{\mathcal{L}\left(\mathbf{L}^{p}(\mathbb{R} ; X)\right)}$.

To exploit the transference principle, we need some condition on the Banach space $X$ that allows us to estimate the norm of the convolution operator $L_{\mu}$, at least in special non-trivial cases. We say that a Banach space $X$ is of class $\mathcal{H} \mathcal{T}_{0}$, if the family of convolution operators $\left(H_{\varepsilon}\right)_{\varepsilon \in(0,1)}$ defined by

$$
\left(H_{\varepsilon} f\right)(t):=\int_{\varepsilon \leq|s| \leq 1} f(t-s) \frac{d s}{s} \quad\left(f \in \mathbf{L}^{2}(\mathbb{R} ; X)\right)
$$

are uniformly bounded in $\mathcal{L}\left(\mathbf{L}^{2}(\mathbb{R} ; X)\right)$. Alternatively, $X$ is of class $\mathcal{H} \mathcal{T}_{0}$ if the limit

$$
H_{0} f:=\lim _{\varepsilon \searrow 0} H_{\varepsilon} f
$$

exists in $\mathbf{L}^{2}(\mathbb{R} ; X)$ for every $f \in \mathbf{L}^{2}(\mathbb{R} ; X)$. The next result shows that this class of Banach spaces is identical to the class of so-called UMD spaces. (See [7, Section E.6] for more information on these class.)

PROPOSITION 3.2. A space $X$ is of class $\mathcal{H} \mathcal{T}_{0}$ if and only if it is a UMD space.

Proof. If $X$ is a UMD space, then define $m_{\varepsilon}:=\mathcal{F}\left(\mathbf{1}_{(\varepsilon \leq|s| \leq 1)} 1 / s\right)$ for $\varepsilon \in(0,1)$. The functions $m_{\varepsilon}$ satisfy Mikhlin's criterion uniformly, i.e.

$$
\sup _{\varepsilon \in(0,1), t \in \mathbb{R}}\left|m_{\varepsilon}(t)\right|+\left|t m_{\varepsilon}^{\prime}(t)\right|<\infty .
$$

Hence by the UMD-version of Mikhlin's theorem [18], the functions $m_{\varepsilon}$ define a uniformly bounded family of $\mathbf{L}^{2}(\mathbb{R} ; X)$-Fourier multipliers. This means that $\sup _{\varepsilon \in(0,1)}\left\|H_{\varepsilon}\right\|<\infty$, i.e. $X$ is of class $\mathcal{H} \mathcal{T}_{0}$.

To prove the converse, suppose that $X$ is of class $\mathcal{H} \mathcal{T}_{0}$. By [2] it suffices to show that $X$ is of class $\mathcal{H} \mathcal{T}$, i.e. the Hilbert transform is bounded on $\mathbf{L}^{2}(\mathbb{R} ; X)$. Consider for $0<\varepsilon<1<T$ the convolution operator

$$
H_{\varepsilon, T} f:=\int_{\varepsilon \leq|s| \leq T} f(t-s) \frac{d s}{s} \quad\left(f \in \mathbf{L}^{2}(\mathbb{R} ; X)\right) .
$$

It suffices to prove that these family of operators is uniformly bounded. But the symbol of $H_{\varepsilon, T}$ is $m_{\varepsilon / T}(t / T)$, and so the assertion follows from the $\mathcal{H} \mathcal{T}_{0}$-property and a general fact on Fourier multipliers [7, Lemma E.4.1, a)]. 
We use the transference principle and the $\mathcal{H} \mathcal{T}_{0}$ (equivalently: UMD) property to obtain information on the functional calculus. To this aim we introduce a certain class of distributions. Let $g \in \mathbf{L}^{1}(-1,1)$ be even, i.e., $g(t)=g(-t)$. We define the distribution $\mathrm{PV}-g(s) / s$ by the formula

$$
\left\langle\mathrm{PV}-\frac{g(s)}{s}, \phi\right\rangle:=\lim _{\varepsilon \searrow 0} \int_{\varepsilon<|s|<1} g(s) \phi(s) \frac{d s}{s}=\int_{0}^{1} g(s) \frac{\phi(s)-\phi(-s)}{s} d s
$$

for $\phi \in \mathcal{D}(\mathbb{R})$. Then it is clear that

$$
\left|\left\langle\mathrm{PV}-\frac{g(s)}{s}, \phi\right\rangle\right| \leq\|g\|_{\mathbf{L}^{1}(-1,1)}\left\|\phi^{\prime}\right\|_{\infty},
$$

whence PV $-g(t) / t$ is in fact a distribution of first order.

LEMMA 3.3. Let $g \in \mathbf{L}^{1}(-1,1)$ be even and define $G:=i(P V-g(s) / s)$. Then the following assertions hold.

a) The distribution $G$ is odd.

b) The Fourier transform of $G$ is given by

$$
\widehat{G}(z)=i \mathrm{PV}-\int_{-1}^{1} g(s) e^{-i s z} \frac{d s}{s}=2 \int_{0}^{1} \frac{\sin (s z)}{s} g(s) d s \quad(z \in \mathbb{C}) .
$$

c) One has $\frac{d}{d z} \widehat{G}(z)=\widehat{g}(z)$ for $z \in \mathbb{C}$, and $\widehat{G}(0)=0$.

The proof is straightforward, so we omit it. Let us consider the special case that $g=\mathbf{1}_{(-1,1)}$

LEMMA 3.4. Let $h:=\mathcal{F}\left(i \mathrm{PV}-\mathbf{1}_{(-1,1)}(s) / s\right)$, i.e.

$$
h(z)=2 \int_{0}^{1} \sin (s z) \frac{d s}{s} \quad(z \in \mathbb{C}) .
$$

Then $h^{\prime}(z)=\mathcal{F}\left(\mathbf{1}_{(-1,1)}\right)=2 \sin z / z, h \in H^{\infty}\left(S t_{\theta}\right)$ and

$$
\lim _{|\operatorname{Im} z|<\theta, \operatorname{Re} z \rightarrow \pm \infty} h(z)= \pm \pi
$$

for every $\theta>0$. 
Proof. Applying Lemma 3.4 with $g=\mathbf{1}_{(-1,1)}$ we obtain

$$
\widehat{g}(z)=\frac{2 \sin z}{z} \quad \text { and } \quad h(z)=\widehat{G}(z)=2 \int_{0}^{z} \frac{\sin w}{w} d w
$$

Fix $\theta>0$. Since

$$
\lim _{t \rightarrow \infty} \int_{0}^{t} \frac{\sin s}{s} d s=\frac{\pi}{2}
$$

the equations (3.1) follow from Cauchy's theorem. A fortiori one has $f \in H^{\infty}\left(\operatorname{St}_{\theta}\right)$.

Given that $-i A$ generates a group on a Banach space $X$ one can now form $h(A)$ by the functional calculus.

PROPOSITION 3.5. Let $-i A$ be the generator of a $C_{0}$-group on a UMD space $X$, and let $h$ be defined as above. Then $h(A) \in \mathcal{L}(X)$ and one has the representation

$$
h(A) x=i P V-\int_{-1}^{1} U(s) x \frac{d s}{s}=i \lim _{\varepsilon \searrow 0} \int_{\varepsilon<|s|<1} U(s) x \frac{d s}{s}
$$

for all $x \in X$. Moreover, the estimate

$$
\sup _{r \in \mathbb{R}}\|h(A+r)\| \leq \sqrt{2} M^{2} c
$$

holds true, where $M:=\sup _{|s| \leq 2}\|U(s)\|$ and $c:=\liminf _{\varepsilon \searrow 0}\left\|H_{\varepsilon}\right\|_{\mathcal{L}\left(\mathbf{L}^{2}(\mathbb{R} ; X)\right)}$.

Proof. Define $g_{\varepsilon}(s)=i \mathbf{1}_{(\varepsilon \leq|s| \leq 1)}(s) / s$ and $h_{\varepsilon}:=\mathcal{F}\left(g_{\varepsilon}\right)$. Then $h_{\varepsilon}(z)=(h(z)-h(\varepsilon z))$ for all $z \in \mathbb{C}$. Hence, fixing $\theta>0$, the family of functions $\left(h_{\varepsilon}\right)_{\varepsilon}$ is uniformly bounded on $S t_{\theta}$ and converges to $h$ uniformly on compact subsets. Let $M:=\sup _{s \in[-2,2]}\|U(s)\|$. Then, by Theorem 3.1,

$$
\left\|h_{\varepsilon}(A+r) x\right\|=\left\|\int_{-1}^{1} h_{\varepsilon}(s) e^{-i r s} U(s) x d s\right\| \leq \sqrt{2} M^{2}\left\|H_{\varepsilon}\right\|_{\mathcal{L}\left(\mathbf{L}^{2}(\mathbb{R} ; X)\right)}\|x\|
$$

for all $x \in X, r \in \mathbb{R}$. Since $X$ is of class $\mathcal{H} \mathcal{T}_{0}, c^{\prime}:=\sup _{\varepsilon}\left\|H_{\varepsilon}\right\|_{\mathcal{L}\left(\mathbf{L}^{2}(\mathbb{R} ; X)\right)}<\infty$. Hence the Convergence Lemma applies and yields

$$
\|f(A+r)\| \leq \sqrt{2} M^{2} c \quad(r \in \mathbb{R}) .
$$


We now use the previous proposition to derive an elegant statement about the functional calculus. Suppose a function $f \in H^{\infty}\left(S t_{\theta}\right)$ can be written as

$$
f=\widehat{g}+c h+d
$$

where $g \in \mathbf{L}_{\omega}^{1}(\mathbb{R})$ and $c, d \in \mathbb{C}$. Then $f$ has limits at $\operatorname{Re} z= \pm \infty$ with

$$
f(\infty)=\pi c+d, \quad f(-\infty)=-\pi c+d .
$$

(This shows that the representation (3.2) is unique.) Furthermore, there exists a function $k$ that is contained in each $\mathbf{L}_{\theta}^{1}(\mathbb{R}), \theta \in[0, \omega)$, such that $f^{\prime}=\widehat{k}$. Indeed, $k=(-i s) g+c \mathbf{1}_{(-1,1)}$.

THEOREM 3.6. Let $-i A$ be the generator of a $C_{0}$-group $U$ on a UMD space $X, \theta>$ $\theta(U)$, and let $f \in H^{\infty}\left(S t_{\theta}\right)$. Suppose that $f$ admits a decomposition

$$
f=\widehat{g}+c h+d
$$

with $g \in \mathbf{L}_{\omega}^{1}(\mathbb{R})$ for some $\omega>\theta(U)$ and $c, d \in \mathbb{C}$. Then

$$
\sup _{r \in \mathbb{R}}\|f(A+r)\|<\infty
$$

and

$$
f(A) x=d x+\lim _{\varepsilon \searrow 0} \int_{|s| \geq \varepsilon} i k(s) U(s) x \frac{d s}{s} \quad(x \in X)
$$

where $\widehat{k}=f^{\prime}$ and $d=[f(\infty)+f(-\infty)] / 2$.

The condition on $f$ is satisfied in particular if $f^{\prime} \in \mathcal{E}(\theta)$.

Proof. The first statement follows directly from the previous proposition. To prove the representation formula (3.4) we fix $x \in X$ and compute

$$
\begin{aligned}
f(A) x & =d x+\widehat{g}(A) x+\operatorname{ch}(A) x=d x+\int_{\mathbb{R}} g(s) U(s) x d s+i c \mathrm{PV}-\int_{-1}^{1} U(s) x \frac{d s}{s} \\
& =d x+\lim _{\varepsilon \searrow 0} \int_{|s| \geq \varepsilon}\left[s g(s)+i c \mathbf{1}_{(-1,1)}\right] U(s) x \frac{d s}{s} \\
& =d x+\lim _{\varepsilon \searrow 0} \int_{|s| \geq \varepsilon} i k(s) U(s) x \frac{d s}{s}
\end{aligned}
$$

where $k(s)=(-i s) g+c \mathbf{1}_{(-1,1)}$. It was observed above that $\widehat{k}=f^{\prime}$. The formula for $d$ is also clear from the above remarks. 

Let

Suppose now that $f^{\prime} \in \mathcal{E}(\theta)$. We apply Lemma 2.2 to find a function $k$ such that $\widehat{k}=f^{\prime}$.

$$
g(s):=\frac{i}{s} k(s) \mathbf{1}_{|s| \geq 1}(s)+i \frac{k(s)-k(0)}{s} \mathbf{1}_{(-1,1)}(s) \quad(s \in \mathbb{R}) .
$$

Since $k$ is Hölder continuous at zero, $g \in \mathbf{L}_{\omega}^{1}(\mathbb{R})$ for all $\omega \in[0, \theta)$. Writing $c:=k(0)$ and recalling that $h^{\prime}=\mathcal{F}\left(\mathbf{1}_{(-1,1)}\right)$, one obtains

$$
(\widehat{g}+c h)^{\prime}=\mathcal{F}((-i s) g)+c h^{\prime}=\mathcal{F}\left((-i s) g+c \mathbf{1}_{(-1,1)}\right)=\widehat{k}=f^{\prime} .
$$

Hence there is a constant $d \in \mathbb{C}$ such that $f=\widehat{g}+c h+d$. This finishes the proof.

EXAMPLE 3.7. The function $f(z)=\arctan z$ is holomorphic and bounded on each strip $S t_{\theta}, \theta<1$. Moreover, $f^{\prime}(z)=\left(1+z^{2}\right)^{-1} \in \mathcal{E}(\theta)$. Hence if $-i A$ generates a $C_{0}$-group on a UMD space $X$ such that $\theta(U)<1$, then $\arctan (A) \in \mathcal{L}(X)$. Moreover, one has the representation

$$
\arctan (A) x=\frac{i}{2} \mathrm{PV}-\int_{\mathbb{R}} e^{-|s|} U(s) x \frac{d s}{s} \quad(x \in X) .
$$

This follows from Theorem 3.6 since

$$
f^{\prime}(z)=\frac{1}{1+z^{2}}=\mathcal{F}\left(\frac{1}{2} e^{-|s|}\right) .
$$

REMARK 3.8. It is known for quite a time that on a UMD space $X$ the principal value integral

$$
\mathrm{PV}-\int_{-1}^{1} \frac{U(s) x}{s} d s
$$

exists for every $x \in X$. This was the key step in Monniaux's paper [13]. Our approach is seemingly a little more involved, but in fact highlights two new things: first, it interprets the result directly in terms of the functional calculus, namely it establishes the identity

$$
\mathrm{PV}-\int_{-1}^{1} \frac{U(s)}{s} d s=\frac{2}{i}\left[\int_{0}^{z} \frac{\sin w}{w} d w\right](A) .
$$

Second, we learn how the different ingredients (transference and UMD-property) work together. This paves the way to more general results on functional calculus. Namely, one can show the following. Let $-i A$ be the generator of a $C_{0}$-group on a UMD space $X$. Then for every $\theta>0, A$ has a bounded $H_{1}^{\infty}$-calculus on $S t_{\theta}$. Here

$$
H_{1}^{\infty}\left(S t_{\theta}\right):=\left\{f \in \mathcal{O}\left(S t_{\theta}\right)|\exists K \geq 0:| f(z)|+| z f^{\prime}(z) \mid \leq K \quad\left(z \in S t_{\theta}\right)\right\} .
$$

See [9] for details. 


\section{Monniaux's Theorem and the Dore-Venni Theorem}

In [13] Monniaux proved the following theorem.

THEOREM 4.1. Let $-i$ A generate a $C_{0}$-group $U$ on a UMD space $X$, such that $\theta(U)<$ $\pi$. Then there exists an injective sectorial operator $B$ on $X$ such that $\log B=A$, i.e., $B^{-i s}=U(s)$ for all $s \in \mathbb{R}$.

Monniaux used the notion of analytic generator of a group to prove Theorem 4.1, but the core ingredient was the boundedness of the $\operatorname{limits}_{\lim _{\varepsilon} \backslash 0} \int_{\varepsilon \leq|s|<1} U(s) x d s / s$. By using our functional calculus framework, we can restate the result: if $-i A$ generates a group $U$ of type $\theta(U)<\pi$ then $B:=e^{A}=\left(e^{z}\right)(A)$ is sectorial, i.e., $(-\infty, 0) \subset \varrho(B)$ and $\sup _{t>0}\left\|t(t+B)^{-1}\right\|<\infty$. (It follows from [7, Lemma 4.2.3, c)] that $e^{A}$ is defined and injective for every strip-type operator $A$ of type less than $\pi$.)

Using Theorem 3.6 we can give a short proof of Monniaux's result. Define

$$
f(z):=\frac{1}{1+e^{z}}
$$

Then, for each $\theta \in(0, \pi), f \in H^{\infty}\left(S t_{\theta}\right)$ and

$$
f^{\prime}(z)=\frac{-e^{z}}{\left(1+e^{z}\right)^{2}} \in \mathcal{E}(\theta)
$$

Since obviously $t\left(t+e^{A}\right)^{-1}=f(A-\log t)$ for all $t>0$,

$$
\sup _{t>0}\left\|t\left(t+e^{A}\right)^{-1}\right\|=\sup _{r \in \mathbb{R}}\|f(A+r)\|<\infty
$$

by Theorem 3.6, and this is the sectoriality of $e^{A}$. Moreover, Theorem 3.6 also yields a representation formula. Namely, $f^{\prime}$ can be written as

$$
f^{\prime}(z)=\frac{-1}{4}\left[\cosh \left(\frac{z}{2}\right)\right]^{-2}=\mathcal{F}\left(\frac{-s}{2 \sinh (\pi s)}\right)
$$

see [14, p.34]. (One can verify this by adapting the arguments from [16, p.81, Example 3].) Moreover $[f(\infty)+f(-\infty)] / 2=(0+1) / 2=1 / 2$. Hence Theorem 3.6 yields

$$
t\left(t+e^{A}\right)^{-1} x=\frac{1}{2} x+\frac{1}{2 i} \mathrm{PV}-\int_{\mathbb{R}} t^{i s} U(s) x \frac{d s}{\sinh (\pi s)} \quad(x \in X, t>0) .
$$

This formula is "classical", cf. [13, Proof of Proposition 3.13] and [15, (3.16)]. 
REMARK 4.2. The characteristic difference between the orginal proof of Monniaux's theorem and ours lies in the fact that we use the language and general results of functional calculus, so that the definition of $e^{A}$ is easy; moreover, with the help of the Convergence Lemma we can go back to a (well known) Fourier transform formula to represent its resolvent. Monniaux has to derive such a formula in the first place, because she talks about analytic generators and it is not at all clear that all that amounts to a Fourier transform in the end.

In [13] Theorem 4.1 is applied to give an alternative proof of the celebrated Dore-Venni theorem. More precisely, Monniaux uses the notion of analytic generator, and proves the theorem in the special case where one of the operators is invertible. We now show how to derive the full Dore-Venni theorem, i.e., including its generalisation due to Prüss and Sohr [15]. Let us begin with making precise what we are talking about.

THEOREM 4.3. (Dore-Venni 1987, Prüss-Sohr 1990)

Let $A, B$ be resolvent-commuting, injective, sectorial operators on a UMD space $X$ such that $A, B \in \mathrm{BIP}$ and $\theta_{A}+\theta_{B}<\pi$. Then there is a constant $K$ such that

$$
\|A x\|+\|B x\| \leq K\|A x+B x\| \quad(x \in \mathcal{D}(A) \cap \mathcal{D}(B)),
$$

i.e., the operator $(A+B, \mathcal{D}(A) \cap \mathcal{D}(B))$ is closed.

For all results and terminology on sectorial operators we refer to [7]. Here $\theta_{A}=$ $\theta(U), \theta_{B}=\theta(V)$, where $U(s)=A^{-i s}, V(s)=B^{i s}$ are the groups of imaginary powers of $A$ and $B$. The main twist in the consideration is to pass from sectorial operators $A, B$ to their $\log$ arithms $A^{\prime}:=\log A$ and $B^{\prime}=-\log B$. Then we have $-i A^{\prime} \sim U$ and $-i B^{\prime} \sim V$. Let us define the product group $W$ by

$$
W(s)=U(s) V(s)=A^{-i s} B^{i s} \quad(s \in \mathbb{R}) .
$$

(Note that the groups $U$ and $V$ commute, whence $W$ is in fact a well defined $C_{0}$-group.) By hypothesis $\theta(W)<\pi$. Let $-i C^{\prime}$ be the generator of $W$ and $C:=e^{C^{\prime}}$. By Monniaux's theorem, $1+C$ is invertible, so that there is a constant $K$ such that

$$
\|x\| \leq K\|(1+C) x\| \quad(x \in \mathcal{D}(C)) .
$$

Suppose that we can prove that

$$
A B^{-1}=e^{A^{\prime}} e^{B^{\prime}} \subset e^{C^{\prime}}=C
$$

in the sense that whenever $x \in \mathcal{R}(\mathcal{B})$ such that $B^{-1} x \in \mathcal{D}(A)$ one has $x \in \mathcal{D}(C)$ and $C x=A B^{-1} x$. In this case we are done, because if $x \in \mathcal{D}(A) \cap \mathcal{D}(B)$ then $B x \in \mathcal{D}\left(A B^{-1}\right)$, and substituting $B x$ for $x$ in (4.1) yields

$$
\|B x\| \leq K\|B x+C B x\|=K\|B x+A x\| .
$$

Symmetry then concludes the argument. 
By elementary semigroup theory, we have $A^{\prime}+B^{\prime} \subset C^{\prime}$. So, if we had defined a joint functional calculus together with appropriate composition rules, the inclusion (4.2) would drop out from general principles. However, in our case we can do it in an elementary way. Let us formulate this in a separate lemma, the proof of which then concludes the proof of Theorem 4.3.

LEMMA 4.4. Let $X$ be any Banach space, and let $U, V$ be commuting $C_{0}$-groups with generators $-i A,-i B$, respectively. Assume that $\theta(U)+\theta(V)<\pi$. If $-i C$ denotes the generator of the product group $W(s)=U(s) V(s), s \in \mathbb{R}$, then

$$
e^{A} e^{B} \subset e^{C}
$$

Proof. By Lemma 2.2 we can choose $g, f \in \mathbf{L}^{1}(\mathbb{R})$ such that $\widehat{g}(z)=e^{z}\left(1+e^{z}\right)^{-3}$ and $\widehat{f}(z)=\left(e^{z}\right)^{2}\left(1+e^{z}\right)^{-3}$. By definition,

$$
e^{A}=\widehat{g}(A)^{-1} \widehat{f}(A),
$$

and likewise for $e^{B}$ and $e^{C}$. Suppose that we can prove that

$$
\widehat{f}(A) \widehat{f}(B) \widehat{g}(C)=\widehat{g}(A) \widehat{g}(B) \widehat{f}(C) .
$$

Then, since all these operators commute with each other, one has

$$
\begin{aligned}
e^{A} e^{B} & =\widehat{g}(A)^{-1} \widehat{f}(A) \widehat{g}(B)^{-1} \widehat{f}(B) \subset \widehat{g}(A)^{-1} \widehat{g}(B)^{-1} \widehat{f}(A) \widehat{f}(B) \\
& =\widehat{g}(A)^{-1} \widehat{g}(B)^{-1} \widehat{g}(C)^{-1} \widehat{g}(C) \widehat{f}(A) \widehat{f}(B) \\
& =\widehat{g}(C)^{-1} \widehat{g}(A)^{-1} \widehat{g}(B)^{-1} \widehat{g}(B) \widehat{g}(A) \widehat{f}(C)=\widehat{g}(C)^{-1} \widehat{f}(C)=e^{C} .
\end{aligned}
$$

To establish (4.3) we use the Phillips calculus, hence it remains to show that

$$
\begin{aligned}
& \iiint g(r) f(t) f(s) U(t+r) V(s+r) d r d s d t \\
& =\iiint f(r) g(t) g(s) U(t+r) V(s+r) d r d s d t .
\end{aligned}
$$

After change of variables this becomes

$$
\iint F(t, s) U(t) V(s) d t d s=\iint G(t, s) U(t) V(s) d t d s,
$$

where

$$
F(t, s):=\int g(r) f(t-r) f(s-r) d r \text { and } G(t, s):=\int f(r) g(t-r) g(s-r) d r .
$$


But taking Fourier transforms yields

$$
\begin{aligned}
& \iint F(t, s) e^{-i(t x+s y)} d t d s \\
& =\iiint g(r) f(t-r) f(s-r) d r e^{-i(t x+s y)} d t d s \\
& =\iiint g(r) f(t) f(s) e^{-i t x} e^{-i s y} e^{-i r(x+y)} d r d t d s \\
& =\widehat{g}(x+y) \widehat{f}(x) \widehat{f}(y)=\widehat{f}(x+y) \widehat{g}(x) \widehat{g}(y) \\
& =\cdots=\iint G(t, s) e^{-i(t x+s y)} d t d s
\end{aligned}
$$

for all $(x, y) \in \mathbb{R}^{2}$, so $F=G$.

REMARK 4.5. The reader may have noticed that in the proof of Lemma 4.4 we essentially use a Phillips joint functional calculus.

\section{Fattorini's Theorem}

Our next application of Theorem 3.6 is a new proof of the celebrated result of Fattorini concerning cosine functions on UMD spaces. It is based on the following proposition.

PROPOSITION 5.1. Let $-i$ A be the generator of a $C_{0}$-group $U$ on a Banach space $X$. If $X$ is a UMD space, then

$$
\mathcal{D}\left(\left(\omega^{2}+A^{2}\right)^{\frac{1}{2}}\right)=\mathcal{D}(A) \quad(\omega>\theta(U)) .
$$

Note that under the hypotheses of the corollary, $\omega^{2}+A^{2}$ is a sectorial operator, see Section 6.3.

Proof. Without loss of generality we may suppose that $\omega=1$. Define

$$
f(z)=\frac{1-i z}{\left(1+z^{2}\right)^{\frac{1}{2}}}=\frac{\left(1+z^{2}\right)^{\frac{1}{2}}}{(1+i z)} .
$$

It suffices to show that $f(A)$ is bounded, because then one can conclude that

$$
\left(1+z^{2}\right)^{\frac{1}{2}}(A)(1+i A)^{-1}=f(A)=(1-i A)\left(1+z^{2}\right)^{-\frac{1}{2}}(A)
$$

is a bounded operator. By a simple composition rule (Proposition 6.3 below) one has $\left(1+z^{2}\right)^{1 / 2}(A)=\left(1+A^{2}\right)^{1 / 2}$, and so $\mathcal{D}\left(\left(1+A^{2}\right)^{1 / 2}\right)=\mathcal{D}(A)$ follows readily. 
To see that $f(A) \in \mathcal{L}(X)$ we note that since

$$
(-2 i) \arctan z=\log \left(\frac{1-i z}{1+i z}\right)
$$

one has

$$
f(z)=\frac{1-i z}{\left(1+z^{2}\right)^{\frac{1}{2}}}=\frac{1-i z}{(1+i z)^{\frac{1}{2}}(1-i z)^{\frac{1}{2}}}=\frac{(1-i z)^{\frac{1}{2}}}{(1+i z)^{\frac{1}{2}}}=\left(\frac{1-i z}{1+i z}\right)^{\frac{1}{2}}=e^{-i \arctan z}
$$

Therefore, $f$ is bounded and $f^{\prime}=$ if $(z)\left(1+z^{2}\right)^{-1} \in \mathcal{E}$. Finally, apply Theorem 3.6.

REMARK 5.2. The previous proposition can also be proved using Example 3.7. In fact, the proof rests on the boundedness of the operator

$$
f(A)=e^{-i \arctan (A)} .
$$

Obviously, this is a consequence of the boundedness of $\arctan (A)$.

For the intended application of Proposition 5.1 we have to make a short digression, referring the reader to [1, Section 3.16] for details. A cosine function on a Banach space $X$ is a strongly continuous mapping $\operatorname{Cos}: \mathbb{R} \longrightarrow \mathcal{L}(X)$ satisfying

$$
\operatorname{Cos}(t-s)+\operatorname{Cos}(t+s)=2 \operatorname{Cos}(t) \operatorname{Cos}(s) \quad(t, s \in \mathbb{R}) .
$$

In particular $\operatorname{Cos}(0)=I, \operatorname{Cos}(t)=\operatorname{Cos}(-t)$, and all operators $\operatorname{Cos}(t), \operatorname{Cos}(s)$ commute with each other. A cosine function $\mathrm{Cos}$ is exponentially bounded, and so its exponential type defined as

$$
\theta(\operatorname{Cos}):=\inf \left\{\theta>0 \mid \exists M \geq 1:\|\operatorname{Cos}(t)\| \leq M e^{\theta|t|}, t \in \mathbb{R}\right\}
$$

is finite. A cosine function Cos has a generator $B$ defined via its resolvent by the Laplace transform formula

$$
\lambda R\left(\lambda^{2}, B\right)=\int_{0}^{\infty} e^{-\lambda t} \operatorname{Cos}(t) d t \quad(\lambda>\theta(\operatorname{Cos})) .
$$

Given a cosine function Cos one defines the associated sine function by

$$
\operatorname{Sin}(t) x:=\int_{0}^{t} \operatorname{Cos}(s) x d s
$$

and then

$$
u(t)=\operatorname{Cos}(t) x+\operatorname{Sin}(t) y
$$


is the (appropriately defined) mild solution to the second-order Cauchy problem

$$
u^{\prime \prime}(t)=B u(t), \quad u(0)=x, u^{\prime}(0)=y
$$

for $x, y \in X$. The cosine and the sine function are linked by the important identity

$$
2 B \operatorname{Sin}(t) \operatorname{Sin}(s)=\operatorname{Cos}(t+s)-\operatorname{Cos}(t-s) \quad(t, s \in \mathbb{R})
$$

and this is indeed an identity, meaning that $\operatorname{Sin}(t) \operatorname{Sin}(s)$ maps $X$ into $\mathcal{D}(B)$.

Associated to a given cosine function is a certain subspace $V$ of the original Banach space $X$, sometimes called the Kisynski space, which can be characterised in two different ways. Originally it arises when one wants to rewrite the second-order problem (5.1) as a system of first-order equations. One is then led to the first order Cauchy problem for the operator

$$
\mathcal{B}:=\left(\begin{array}{ll}
0 & 1 \\
B & 0
\end{array}\right)
$$

on the product space $X \times X$. However, in general this operator does not generate a semigroup but only a so-called once integrated group, given by

$$
\mathcal{S}(t):=\left(\begin{array}{cc}
\operatorname{Sin}(t) & \int_{0}^{t} \operatorname{Sin}(s) d s \\
\operatorname{Cos}(t)-I & \operatorname{Sin}(t)
\end{array}\right) \quad(t \in \mathbb{R}) .
$$

By general theory of once integrated semigroups, there is a subspace $\mathcal{V} \subset X \times X$ such that $\mathcal{D}(B) \times X \subset \mathcal{V}$ and the part of $\mathcal{B}$ in $\mathcal{V}$ generates a $C_{0}$-group. Kisynski proved in [11] that a space with this property is unique, and that it is of the form $\mathcal{V}=V \times X$, where

$$
V:=\{x \in X \mid \operatorname{Sin}(\cdot) x \in \mathbf{C}([0,1] ; \mathcal{D}(B))\}
$$

with the norm $\|x\|_{V} \sim\|x\|_{X}+\|B \operatorname{Sin}(t) x\|_{\mathbf{C}([0,1] ; X)}$. The group generated by $\mathcal{B}$ on $\mathcal{V}$ is then

$$
\mathcal{U}(t):=\left(\begin{array}{cc}
\operatorname{Cos}(t) & \operatorname{Sin}(t) \\
B \operatorname{Sin}(t) & \operatorname{Cos}(t)
\end{array}\right) \quad(t \in \mathbb{R}) .
$$

The space $\mathcal{V}=V \times X$ is called the phase space of the cosine function, and it is of fundamental importance, in particular to obtain viable perturbation theorems. It is therefore desirable to have a description of $V$ or $\mathcal{V}$ that is not in terms of the cosine function (i.e. the solutions of (5.1)) but in terms of the generator $B$ directly. Such a description was given by Fattorini in [5], cf. also [1, Theorem 3.16.7]. 
THEOREM 5.3. (Fattorini 1969)

Let $B$ generate a cosine function $C o s$ on a Banach space $X$, and let $V$ be its Kisynski space. If $X$ is a UMD space then, for arbitrary $\omega>\theta(\mathrm{Cos})$,

$$
V=\mathcal{D}\left(\left(\omega^{2}-B\right)^{\frac{1}{2}}\right)
$$

Moreover, the operator $-i\left(\omega^{2}-B\right)^{1 / 2}$ generates a $C_{0}$-group on $X$.

One can easily prove that for $\omega>\theta(\operatorname{Cos})$ the operator $\omega^{2}-B$ is invertible and sectorial, and so the square root makes sense.

To prove Theorem 5.3 we will apply Proposition 5.1 to the group $\mathcal{U}$ generated by $\mathcal{B}$ on $\mathcal{X}:=V \times X$. Clearly, without loss of generality we may suppose that $\theta(\mathrm{Cos})<1=\omega$. Define $\mathcal{A}:=i \mathcal{B}$, so that $-i \mathcal{A}=\mathcal{B}$ generates $\mathcal{U}$. By Lemma 6.1 and Theorem 6.2 below, we have

1) $\mathcal{V}$ is a UMD space.

2) $\theta(\mathcal{U})<1$

So by Proposition 5.1 one has

$$
\mathcal{D}(\mathcal{A})=\mathcal{D}\left(\left(1+\mathcal{A}^{2}\right)^{\frac{1}{2}}\right) .
$$

Now, $\mathcal{D}(\mathcal{A})=\mathcal{D}(B) \times V$ and

$$
\mathcal{A}^{2}=\left(\begin{array}{cc}
-B & 0 \\
0 & -B
\end{array}\right), \quad \mathcal{D}\left(\mathcal{A}^{2}\right)=\mathcal{D}\left(\left.B\right|_{V}\right) \times \mathcal{D}(B),
$$

whence clearly

$$
\left(1+\mathcal{A}^{2}\right)^{1 / 2}=\left(\begin{array}{cc}
(1-B)^{1 / 2} & 0 \\
0 & (1-B)^{1 / 2}
\end{array}\right)
$$

with

$$
\mathcal{D}\left(\left(1+\mathcal{A}^{2}\right)^{1 / 2}\right)=\mathcal{D}\left(\left.(1-B)^{1 / 2}\right|_{V}\right) \times \mathcal{D}\left((1-B)^{1 / 2}\right) .
$$

Looking at the second component we arrive at

$$
V=\mathcal{D}\left((1-B)^{1 / 2}\right)
$$

as we wanted to prove. The rest of Fattorini's theorem is easy: the matrix

$$
T:=\left(\begin{array}{cc}
-i(1-B)^{-1 / 2} & 0 \\
0 & 1
\end{array}\right)\left(\begin{array}{cc}
1 & -1 \\
1 & 1
\end{array}\right): X \times X \longrightarrow V \times X
$$


is an isomorphism with inverse

$$
T^{-1}=\frac{1}{2}\left(\begin{array}{cc}
1 & 1 \\
-1 & 1
\end{array}\right)\left(\begin{array}{cc}
i(1-B)^{1 / 2} & 0 \\
0 & 1
\end{array}\right) .
$$

The isomorphism $T$ maps $V \times V$ onto $\mathcal{D}\left(\left.(1-B)^{1 / 2}\right|_{V}\right) \times \mathcal{D}\left((1-B)^{1 / 2}\right)$. Hence

$$
T^{-1}\left(\begin{array}{cc}
0 & 1 \\
-(1-B) & 0
\end{array}\right) T=\left(\begin{array}{cc}
i(1-B)^{1 / 2} & 0 \\
0 & -i(1-B)^{1 / 2}
\end{array}\right) .
$$

But

$$
\left(\begin{array}{cc}
0 & 1 \\
-(1-B) & 0
\end{array}\right)=\left(\begin{array}{ll}
0 & 1 \\
B & 0
\end{array}\right)-\left(\begin{array}{ll}
0 & 0 \\
1 & 0
\end{array}\right)
$$

is a bounded perturbation of the generator of a group, whence is itself a group generator. Hence, by similarity, $\operatorname{diag}\left(i(1-B)^{1 / 2},-i(1-B)^{1 / 2}\right)$ generates a group on $X \times X$. Taking the first (or second) component concludes the proof of Theorem 5.3.

Let us conclude this section with the standard counterexample.

COUNTEREXAMPLE 5.4. We consider the shift group $U$ on the space $X=\mathbf{L}^{1}(\mathbb{R})$. Then $\operatorname{Cos}(t):=(1 / 2)(U(t)+U(-t))$ defines a bounded cosine function. The generator of $\operatorname{Cos}$ is the second derivative, i.e. the one-dimensional Laplacian $\Delta$. The Kisynski space $V$ equals the domain of the generator of the group $U$, i.e., $V=\mathcal{D}(d / d t)=\mathbf{W}^{\mathbf{1}, \mathbf{1}}(\mathbb{R})$. But

$$
V=\mathbf{W}^{\mathbf{1}, \mathbf{1}}(\mathbb{R}) \neq \mathcal{D}\left((1-\Delta)^{1 / 2}\right),
$$

otherwise $z\left(1+z^{2}\right)^{-1 / 2}$ would be a Fourier multiplier on $\mathbf{L}^{1}(\mathbb{R})$, which is not because $\lim _{t \rightarrow \pm \infty} t\left(1+t^{2}\right)^{-1 / 2}= \pm 1$.

\section{Appendix: Missing Facts}

In this appendix we provide three facts that were needed in the main part of the paper.

\subsection{The Kisynski space is UMD}

Let $B$ generate a cosine function Cos on the Banach space $X$, and let Sin be the associated sine function. The Kisynski space $V$ is given by

$$
V:=\{x \in X \mid B \operatorname{Sin}(t) x \in \mathbf{C}([0,1] ; X)\}
$$

with the norm $\|x\|_{V}:=\|x\|+\sup _{t \in[0,1]}\|B \operatorname{Sin}(t) x\|_{X}$. If $X$ is a UMD space/Hilbert space then $V$ is so as well, but up to now this was an a posteriori result following from Fattorini's theorem. Since our proof of Fattorini's theorem uses this fact, we need to provide a different argument. The result will follow from the next lemma. 
LEMMA 6.1. In the situation described above, the norm

$$
\|x\|:=\left(\|x\|_{X}^{2}+\|B \operatorname{Sin}(t)\|_{\mathbf{L}^{2}([0,1] ; X)}^{2}\right)^{\frac{1}{2}}
$$

is an equivalent norm on $V$.

Proof. The inequality $\|x\| \leq \sqrt{2}\|x\|_{V}$ is trivial. For the converse inequality we note first that from (5.2) it follows that there is $M \geq 0$ such that

$$
\sup _{t \in[0,1]}\|\operatorname{Sin}(t) y\|_{V} \leq M\|y\|_{X} \quad(y \in V) .
$$

Using this and (5.2) again, we estimate

$$
\begin{aligned}
\|x\|_{V} & =\left\|\frac{1}{2} \int_{0}^{2}(x-\operatorname{Cos}(t) x) d t+\frac{1}{2} \operatorname{Sin}(2) x\right\|_{V} \\
& \leq\left\|\int_{0}^{1}(x-\operatorname{Cos}(2 t) x) d t\right\|_{V}+\|\operatorname{Cos}(1) \operatorname{Sin}(1) x\|_{V} \\
& =\left\|\int_{0}^{1}-2 B \operatorname{Sin}(t) \operatorname{Sin}(t) x d t\right\|_{V}+\|\operatorname{Cos}(1) \operatorname{Sin}(1) x\|_{V} \\
& \leq 2 M \int_{0}^{1}\|B \operatorname{Sin}(t) x\|_{X} d t+M\|\operatorname{Cos}(1) x\|_{X} \\
& \leq(2+\|\operatorname{Cos}(1)\|) M\left(\|x\|_{X}+\|B \operatorname{Sin}(t) x\|_{\mathbf{L}^{1}([0,1] ; X)}\right) \\
& \leq \sqrt{2}(2+\|\operatorname{Cos}(1)\|) M\|x\|
\end{aligned}
$$

(This proof shows that actually all norms $\|x\|_{p}=\left(\|x\|_{X}^{p}+\|B \operatorname{Sin}(t) x\|_{\mathbf{L}^{p}([0,1] ; X)}^{p}\right)^{1 / p}$ on $V$ are equivalent.)

\subsection{The type of the induced group}

In our proof of Fattorini's theorem we used the fact that when passing from a cosine function Cos to the group $\mathcal{U}$ on the phase space $V \times X$ the exponential growth type does not change. Now, it is easy to see that at least the once integrated group $(\mathcal{S}(t))_{t \in \mathbb{R}}$ given by (5.3) does not have a bigger growth type than the original cosine function Cos. To be more precise, if $\|\operatorname{Cos} t\| \leq M e^{\omega|t|}$, then for each $\varepsilon>0$ we can find $M_{\varepsilon}$ such that $\|\mathcal{S}(t)\| \leq M_{\varepsilon} e^{(\omega+\varepsilon)|t|}$. The group $\mathcal{U}$ arises by taking the part of the generator of $\mathcal{S}$ in a certain subspace, and this comes 
entirely from the theory of integrated semigroups. Unfortunately, the standard reference [1, Theorem 3.10.4] deals only with semigroups here, but we need a two-sided estimate, hence we cannot just cite the literature.

THEOREM 6.2. Let $A$ be the generator of a once integrated group $(S(t))_{t \in \mathbb{R}}$ on a Banach space $X$ such that $\|S(t)\| \leq M e^{\omega|t|}$. Then for each $\omega^{\prime}>\omega$ there exists a subspace $V$ of $X$ such that $\mathcal{D}(A) \subset V$ and the part of $A$ in $V$ generates a group $U$ of growth type $\theta(U)<\omega^{\prime}$.

Observe that in the general situation of the theorem, the space $V$ will differ as we make $\omega^{\prime}$ smaller. However, when dealing with cosine functions, each of these spaces will coincide with the phase space, by Kisynski's theorem.

Proof. We modify the proof of [1, Theorem 3.10.4] in the case $k=1$ to the setting of groups. To be able to write expressions like $A S(t)$ without domain restrictions, we shall freely use the framework of the universal extrapolation space, say $Y \supset X$, (as in [7, Section 6.3.1]). This means that e.g. $y=A S(t) x \in Y$ is defined for all $x \in Y$, but if $x \in X$ one does not necessarily have $A S(t) x \in X$ again. The space $Y$ is a mere vector space, but is the union of an increasing sequence of Banach spaces $\left(X_{-n}\right)_{n \in \mathbb{N}}$, with $X_{0}=X$. The operator $A$ can be extended to a fully defined operator on $Y$, mapping each $X_{-n}$ into $X_{-n+1}$. Since the group $U$ is supposed to be such that $S(t)=\int_{0}^{t} U(s) d s$, we define

$$
U(t):=\left\{\begin{array}{ll}
A S(t)+I, & t \geq 0 \\
-A S(t)+I, & t \leq 0
\end{array}=\operatorname{sgn}(t) A S(t)+I \quad(t \in \mathbb{R}) .\right.
$$

To prove that $U$ is indeed a group, we take Laplace transforms within $Y$

$$
R_{\lambda} x:=\int_{0}^{\infty} e^{-\lambda t} U(t) x d t=R(\lambda, A) x \quad(\lambda>\omega, x \in Y)
$$

where the integral is actually taken within $X_{-n}$ with $n$ large enough such that $A x \in X_{-n}$. By the very definition of $A$ (and its extension to $Y$ ) it follows that $R_{\lambda}=R(\lambda, A)$. Likewise,

$$
\int_{0}^{\infty} e^{-\lambda t} U(-t) d t=R(\lambda,-A) \quad(\lambda>\omega)
$$

Now the arguments of [1, Theorem 3.1.7] can be adapted to show that $U$ is in fact a group on the universal extrapolation space $Y$ (cf. also [1, Proposition 3.1.18]). Define

$$
\|x\|_{W}:=\sup _{t \in \mathbb{R}}\left\|e^{-\omega^{\prime}|t|} U(t) x\right\|_{X}
$$


and $W:=\left\{x \mid\|x\|_{W}<\infty\right\}$. It is clear that $W \subset X$ with contractive inclusion, and that $W$ is a Banach space with that norm. A short computation shows that $W$ is invariant under the group $U$ and one has

$$
\|U(t) x\|_{W} \leq e^{\omega^{\prime}|t|}\|x\|_{W} \quad(x \in W, t \in \mathbb{R}) .
$$

Furthermore, $\mathcal{D}(A) \subset W$ since for $x \in \mathcal{D}(A)$ we have

$$
\left\|e^{-\omega|t|} U(t) x\right\|_{X} \leq\left\|e^{-\omega|t|} A S(t) x\right\|+\left\|e^{-\omega|t|} x\right\|_{X} \leq M\|A x\|_{X}+\|x\|_{X} .
$$

Finally, $\|R(\lambda, A)\|_{W \rightarrow W} \leq\|R(\lambda, A)\|_{X \rightarrow X}$, for every $\lambda \in \varrho(A)$. In particular,

$$
\sup _{\lambda>\omega}\|\lambda R(\lambda, A)\|_{W \rightarrow W}<\infty .
$$

Define $V$ to be the closure of $\mathcal{D}(A)$ in $W$. It remains to show that $V$ is $U$-invariant and $U$ is strongly continuous on $V$. For this it suffices to show that

$$
\|\lambda R(\lambda, A) x-x\|_{W} \rightarrow 0 \quad \text { as } \quad \lambda \rightarrow \infty
$$

for each $x \in \mathcal{D}(A)$. So fix $x \in \mathcal{D}(A)$ and $\varepsilon>0$. Then for $\lambda \geq 2 \omega$

$$
\begin{aligned}
\left\|e^{-\omega^{\prime}|t|} U(t)(\lambda R(\lambda, A) x-x)\right\|_{X} \\
=\left\|e^{-\omega^{\prime}|t|} \int_{0}^{\infty} \lambda e^{-\lambda s}(U(t+s) x-U(t) x) d s\right\| \\
=\left\|e^{-\omega^{\prime}|t|} \int_{0}^{\infty} \lambda e^{-\lambda s}(\operatorname{sgn}(s+t) S(t+s) A x-\operatorname{sgn}(t) S(t) A x) d s\right\| \\
\leq M e^{-\left(\omega^{\prime}-\omega\right)|t|} \frac{2 \lambda-\omega}{\lambda-\omega}\|A x\| \leq 3 M e^{-\left(\omega^{\prime}-\omega\right)|t|}\|A x\|<\varepsilon
\end{aligned}
$$

for all $t$ such that $|t| \geq T$, for some $T>0$. On the other hand, for some $\tau>0$

$$
\|\operatorname{sgn}(s+t) S(s+t) A x-\operatorname{sgn}(t) S(t) A x\| \leq \varepsilon \quad(|t| \leq T, s \in[0, \tau]) .
$$

This is due to the fact that $S(0)=0$ and therefore $t \longmapsto \operatorname{sgn}(t) S(t)$ is strongly continuous on $\mathbb{R}$. Hence

$$
\begin{aligned}
& \left\|e^{-\omega^{\prime}|t|} U(t)(\lambda R(\lambda, A) x-x)\right\|_{X} \\
& \quad \leq \int_{0}^{\tau} \lambda e^{-\lambda s} \varepsilon d s+M e^{-\left(\omega^{\prime}-\omega\right)|t|} \int_{\tau}^{\infty} \lambda e^{-\lambda s}\left(1+e^{\omega s}\right) d s \\
& \quad=\varepsilon+M e^{-\lambda \tau}\left(1+\frac{\lambda}{\lambda-\omega} e^{\omega \tau}\right) \leq \varepsilon+M\left(1+2 e^{\omega \tau}\right) e^{-\lambda \tau} \leq 2 \varepsilon
\end{aligned}
$$

for large $\lambda>2 \omega$. 


\subsection{A composition rule}

We use the terminology of [7]. For a strong strip-type operator $A$ denote by $\omega_{s s t}(A)$ the infimum of all $\omega>0$ such that there is a constant $M_{\omega}$ with

$$
\|R(\lambda, A)\| \leq M_{\omega}(|\operatorname{Im} \lambda|-\omega)^{-1} \quad(|\operatorname{Im} \lambda|>\omega)
$$

Then, if $A$ is an operator is such that $-i A$ generates a group $U, A$ is strong strip-type with $\omega_{s s t}(A) \leq \theta(U)$. The following statement is certainly not optimal, but serves our purposes.

PROPOSITION 6.3. Let A be a strong strip-type operator on a Banach space X. Then for all $\omega>\omega_{\text {sst }}(A)$ the operator $B:=\omega^{2}+A^{2}$ is invertible and sectorial of angle $\omega_{\text {sect }}(B)<\pi / 2$. Moreover, let $f$ be holomorphic on $(\operatorname{Re} z>0)$ such that $f(B)$ is defined by the natural functional calculus for invertible sectorial operators. Then $\left[f\left(\omega^{2}+z^{2}\right)\right](A)$ is defined by the functional calculus for A, and one has

$$
\left[f\left(\omega^{2}+z^{2}\right)\right](A)=f\left(\omega^{2}+A^{2}\right) .
$$

Proof. The hypothesis implies that there is $\alpha \in(0, \omega)$ and $M \geq 0$ such that

$$
\|R(\lambda, A)\| \leq M(|\operatorname{Im} \lambda|-\alpha)^{-1} \quad(|\operatorname{Im} \lambda|>\alpha) .
$$

Take $\mu \in \mathbb{C}$ such that $\operatorname{Re} \mu \leq 0$. Then there is $\lambda=x+i y$ such that $\operatorname{Im} \lambda=y \geq \omega$ and $\lambda^{2}=\mu-\omega^{2}$. Hence

$$
\mu R(\mu, B)=\mu R\left(\mu, \omega^{2}+A^{2}\right)=\mu R\left(\lambda^{2}, A^{2}\right)=-\left(\lambda^{2}+\omega^{2}\right) R(\lambda, A) R(-\lambda, A) .
$$

Estimating yields

$$
\|\mu R(\mu, B)\| \leq M^{2} \frac{|\lambda|^{2}+\omega^{2}}{(\operatorname{Im} \lambda-\alpha)^{2}}=M^{2} \frac{x^{2}+y^{2}+\omega^{2}}{(y-\alpha)^{2}} .
$$

But $x^{2}-y^{2}+\omega^{2}=\operatorname{Re} \mu \leq 0$ and hence

$$
\|\mu R(\mu, B)\| \leq 2 M^{2}\left(\frac{y}{y-\alpha}\right)^{2} .
$$

which is uniformly bounded in $y \geq \omega$. So indeed $B=\omega^{2}+A^{2}$ is invertible and sectorial of angle $<\pi / 2$.

The proof of the composition rule is fairly standard. One uses [7, Proposition 1.3.6] to reduce everything to the case that $f$ is an elementary (= well behaved) function. For such $f$ one employs arguments similar to those in [7, Lemma 2.4.4] or [7, Theorem 4.2.4]. 


\section{Acknowledgement}

The author wants to thank Wolfgang Arendt for a quite motivating conversation on a free afternoon at Newcastle, and Bernhard Haak for ongoing discussions and continuous, very fruitful and critical questions and remarks. Last, the author thanks the referee for his careful reading of the paper, and for a nice remark that led to a considerable improvement.

\section{REFERENCES}

[1] ARendt, Wolfgang and BAtTy, J. K. Charles, Matthias Hieber, and Frank Neubrander, Vector-Valued Laplace Transforms and Cauchy Problems, Monographs in Mathematics 96, Basel: Birkhäuser, xi, 2001, p. 523.

[2] BouRgaIn, J., Some remarks on Banach spaces in which martingale difference sequences are unconditional, Ark. Mat. 21(2) (1983), 163-168.

[3] BoyadzhieV, KHRISTO and DeLAubenfels, RALPH, Spectral theorem for unbounded strongly continuous groups on a Hilbert space, Proc. Amer. Math. Soc. 120(1) (1994), 127-136.

[4] Dore, Giovanni and Venni, Alberto, On the closedness of the sum of two closed operators, Math. Z. 196(2) (1987), 189-201.

[5] FATTORINI, H. O., Ordinary differential equations in linear topological spaces. II, J. Differential Equations 6 (1969), 50-70.

[6] HAASE, MARKUS, Spectral properties of operator logarithms, Math. Z. 245(4) (2003), 761-779.

[7] HAASE, MARKuS The Functional Calculus for Sectorial Operators, Number 169 in Operator Theory: Advances and Applications, Birkhäuser-Verlag, Basel, 2006.

[8] HAASE, MARKUS, Semigroup theory via functional calculus, Preprint, 2006.

[9] HAASE, MARKUS, A transference principle for general groups and functional calculus on UMD spaces, Submitted, 2006.

[10] Kalton, N. J. and Weis, L., The $H^{\infty}$-calculus and sums of closed operators, Math. Ann. 321(2) (2001), 319-345.

[11] KISYŃSKI, J., On cosine operator functions and one-parameter groups of operators, Studia Math. 44 (1972), 93-105. Collection of articles honoring the completion by Antoni Zygmund of 50 years of scientific activity. I.

[12] MCINTOSH, ALAN, Operators which have an $H_{\infty}$ functional calculus, In Miniconference on operator theory and partial differential equations (North Ryde, 1986), Austral. Nat. Univ., Canberra, 1986, pp. 210-231.

[13] MonniauX, SYlviE, A new approach to the Dore-Venni theorem, Math. Nachr. 204 (1999), 163-183.

[14] OBERHETTINGER, Fritz, Tabellen zur Fourier Transformation, Springer-Verlag, Berlin, 1957.

[15] PRUSS, JAN and SOHR, HERMANN, On operators with bounded imaginary powers in Banach spaces, Math. Z. 203(3) (1990), 429-452.

[16] Stein, M. Elias and ShaKarChi, Rami, Complex analysis, Princeton Lectures in Analysis, II. Princeton University Press, Princeton, NJ, 2003.

[17] UITERDIJK, MARC, A functional calculus for analytic generators of $C_{0}$-groups, Integral Equations Operator Theory 36(3) (2000), 349-369.

[18] ZIMMERMANN, FRANK, On vector-valued Fourier multiplier theorems, Studia Math. 93(3) (1989), $201-222$.

Markus Haase

Department of Applied Mathematics

Technical University of Delft

PO Box 5031, 2600 GA Delft

The Netherlands

m.h.a.haase@tudelft.nl 